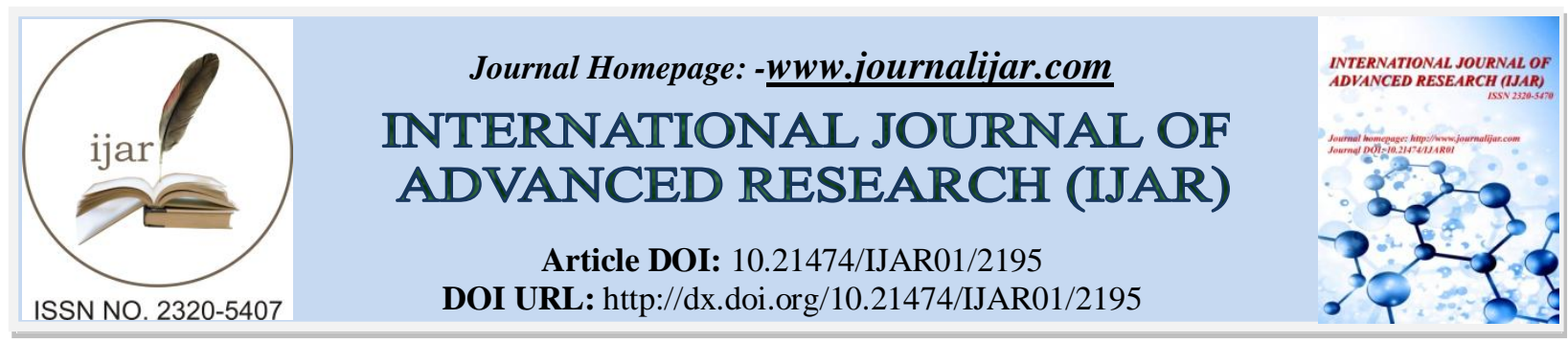

RESEARCH ARTICLE

\title{
FOR A STRESS AMONG SURGERY RESIDENT AND INTERN IN KSA, TO FIND OUT IF THEY SUFFER FROM STRESS OR NOT AT THE WORK PLACE.
}

Aisha Nasser Aqahtani and Zainah Nasser Alqahtani.

\section{Manuscript Info}

Manuscript History

Received: 27 September 2016

Final Accepted: 30 October 2016

Published: November 2016
Abstract

Copy Right, IJAR, 2016,. All rights reserved.

\section{Introduction:-}

Prevalence stress in Surgery resident and intern

There have been many attempts to define stress and burnout. Stress is generally accepted to be "the non-specific physiological changes that occur in response to noxious stimuli". According to The Health \& Safety Executive, stress is defined as "the reaction people have to excessive pressures or other types of demands placed upon them". Burnout, as Merriam-Webster defines it, is "exhaustion of physical or emotional strength or motivation usually as a result of prolonged stress or frustration". Therefore, burnout is caused by stress, and is characterized by three syndromes: emotional exhaustion, depersonalization, and dissatisfaction with one's work.

Pakistan American Medical News reported the findings of a survey that included A total of 133 residents responded to our questionnaire. $74 \%$ respondents were male and $26 \%$ were female. All residents showed a very high level of burnout. 50.4\% residents had high levels of burn out on the EE category, $49.6 \%$ in the DP category and 53.4\% residents had low levels on the PA scale. When assessed for overall burn out $57.9 \%$ residents had overall burnout. Males were more prone to develop burnout. Job dissatisfaction was the most prevalent reason for burn out. Owning a smartphone was significantly associated with development of burnout. Awareness regarding the burnout syndrome and having children were two factors which had a protective effect against burnout syndrome. Marriage, working hours, financial conditions, smoking and consumption of coffee/tea had no effect on development of burnout syndrome.

\section{Research objectives:- \\ TO MUESURE the prevalence OF STRESS AMONG SURGERY RESIDENT AND INTERN. \\ TO explore THE CAUSES OF STRESS SURGERY RESIDENT AND INTERN. \\ TO explore THE MOTHODS USED TO RELEAVE THE STRESS.}

\section{Literature Review:-}

Burnout among surgical residents in a lower-middle income country - Are we any different? In Pakistan

A total of 133 residents responded to our questionnaire. $74 \%$ respondents were male and $26 \%$ were female. All residents showed a very high level of burnout. 50.4\% residents had high levels of burn out on the EE category, $49.6 \%$ in the DP category and $53.4 \%$ residents had low levels on the PA scale. When assessed for overall burn out $57.9 \%$ residents had overall burnout. Males were more prone to develop burnout. Job dissatisfaction was the most prevalent reason for burn out. Owning a smartphone was significantly associated with development of burnout. Awareness regarding the burnout syndrome and having children were two factors which had a protective effect 
against burnout syndrome. Marriage, working hours, financial conditions, smoking and consumption of coffee/tea had no effect on development of burnout syndrome.

A Values Affirmation Intervention to Improve Female Residents' Surgical PerformanceFemale residents in surgical training may face stereotype threat. The awareness of negative stereotypes about surgical ability based on gender may heighten stress and thus reduce performance.This is a randomized, controlled trial in which 167 residents were invited to participate. A total of 45 resident volunteers, including 18 women, were randomized to the affirmation condition or the no-affirmation condition. We administered a values affirmation intervention and measured clinical evaluations data both prior to and 6 months after the intervention. Women benefited from the affirmation. Women who had participated in the affirmation exercise earned higher clinical evaluation scores than those in the control condition $(\mathrm{B}=0.34, \mathrm{P}<.05)$. For men, performance did not differ by affirmation condition $(\mathrm{B}=-0.20, \mathrm{P}=.35)$.

In a large study5 of 582 surgeons who trained at the University of Michigan-Ann Arbor, 32\% showed high levels of emotional exhaustion, $13 \%$ showed high levels of depersonalization, and $4 \%$ showed evidence of a low sense of personal accomplishment. Notably, younger surgeons were more susceptible to burnout than their older colleagues $(\mathrm{P}<.01)$. Burnout was not found to be related to caseload, practice setting, or percentage of patients insured by a health maintenance organization. In contrast, etiologic factors associated with burnout were a sense that work was "overwhelming"; a perceived imbalance among career, family, and personal growth; perceptions that one's career is unrewarding; and lack of autonomy. More important, burnout was strongly associated with a desire to pursue early retirement.

In the recently published survey7 of 549 members of the Society of Surgical Oncology, $28 \%$ of respondents met the criteria for burnout. In addition, approximately $30 \%$ of study participants screened positive for depression. Given the sensitivity (96\%) and specificity (57\%) of the screening instrument used,41,43 this finding implies that approximately $10 \%$ of respondents would have met the criteria for major depressive disorder at the time of the survey if they had undergone a full psychiatric assessment. Burnout was more common among respondents 50 years or younger $(31 \%$ vs $22 \% ; \mathrm{P}=.03)$ and women $(37 \%$ vs $26 \% ; \mathrm{P}=.03)$. Burnout was strongly associated with both potentially problematic alcohol use $(12.4 \%$ burned out vs $4.6 \%$ not burned out; $\mathrm{P}=.001)$ and lower career satisfaction. No differences were found between private practice physicians and academic physicians in frequency of burnout, symptoms of depression, potentially problematic alcohol or other drug use, or mental or physical quality of life.

These issues are not unique to US surgeons. An Australian study9 of 126 surgeons indicated that burnout levels were significantly higher for surgeons than for the normative population, with $47.6 \%$ of the sample reporting high burnout levels. Younger surgeons reported significantly higher burnout levels, regardless of career stage. Burnout correlated significantly with early retirement and/or intentions for retraining and was inversely related to overall emotional intelligence levels. Another study10 of 501 colorectal and vascular surgeons in the United Kingdom showed that $32 \%$ had high burnout on at least 1 subscale of the Maslach Burnout Inventory. Surgeons who planned to take early retirement or wished to retire as soon as they could afford to do so were more likely to have psychiatric morbidity and/or burnout.

\section{Head and Neck Surgeons:-}

Johnson and colleagues 11 conducted a survey of 395 members of the American Society of Head and Neck Surgery and the Society of Head and Neck Surgeons in 1993. A total of 34\% who responded believed they were "burned out." Most respondents replied that they enjoyed their work, but the stress of extended work hours, dealing with seriously ill patients, and the increased need to deal with governmental and economic issues were their sources of greatest concern.

\section{Transplantation Surgeons:-}

Bertges and colleagues 8 conducted a survey of 209 actively practicing transplantation surgeons. Burnout was present in $38 \%$ of respondents. Several significant predictors of emotional exhaustion were identified and included questioning one's career choice, giving up outside activities, and perceiving oneself as having limited control over the provision of medical services. In contrast, surgeons who reported greater professional growth opportunities, institutional support, and a sense of being appreciated by their patients experienced a high sense of personal accomplishment. Prioritizing goals to reflect both personal and professional values was associated with both lower emotional exhaustion and a higher sense of personal accomplishment. 
DIVOOne large, prospective study26 of 1118 married physicians found that the cumulative incidence of divorce after 30 years of marriage was $29 \%$. Specialty choice was independently associated with the risk of divorce, with the highest divorce rates among surgeons (33\% after 30 years) and psychiatrists (50\% after 30 years).26 Higher levels of anger and getting married before medical school graduation were also associated with higher divorce rates.26 Surgeons continued to have a higher divorce rate (relative risk of 1.7 compared with internal medicine physicians) on multivariate analysis controlling for other factors.26 Several studies27- 30 of physicians and their spouses suggest that the ways in which couples treat each other, communication styles, differences in the need for intimacy, and the degree of work-related stress are more important factors for marital satisfaction than the number of hours worked by physicians.RCE AMONG PHYSICIANS AND SURGEONS.

DEPRESSION AND SUICIDEIn a prospective study of more than 1300 male physicians from The Johns Hopkins University, the lifetime prevalence of clinically significant depression was $12.8 \% .13$ In a study of 4500 female physicians in the Women Physicians' Health Study, the prevalence was 19.5\%.14,15 In another study,16 20\% of nearly 2000 survey respondents who are full-time academic physicians and basic science faculty members had significant levels of depressive symptoms, with higher levels in younger faculty members. Although the lifetime rates of depression among physicians appear to be similar to those of the general population, suicide is a disproportionately high cause of mortality in physicians. 17 Compared with other professionals, a male physician's proportionate mortality ratio from suicide is nearly 1.5 - to 3.8 -fold higher . The discrepancy is even greater among female physicians, who have a 3.7 - to 4.5 -fold increased risk of death from suicide.

ALCOHOL AND SUBSTANCE ABUSEPhysician impairment by substance abuse represents a significant challenge with potential grave consequences for patients and society. Although data are sparse, the prevalence of alcohol and illicit drug abuse among physicians is probably similar to that of the general population.20- 24 Problematic alcohol use (ie, symptoms of alcohol dependence or having had $\geq 6$ drinks on 1 occasion in the last year) was reported by $6.8 \%$ of surgeons in a recent study 7 of members of the Society of Surgical Oncology, with a similar frequency reported in a separate study6 of 110 surgical residency graduates from the University of WisconsinMadison. Most physicians who are treated for alcoholism or drug dependence appear to have favorable outcomes, 25 although potential ramifications for licensing and hospital privileges may deter some physicians from seeking assistance.

WHAT ARE THE CAUSES OF BURNOUT One of the tragic paradoxes of burnout is that those most susceptible appear to be the most dedicated, conscientious, responsible, and motivated. Individuals with these traits are often idealistic and have perfectionist qualities that may lead them to submerse themselves in their work and devote themselves to it until they have nothing left to give. Thus, commitment to patients, attention to detail, and recognizing the responsibility associated with patients' trust—-the very traits that define a good surgeon—also place surgeons with these qualities at greater risk for burnout.4,44

A number of studies32- 35,42 have explored the potential causes of physician burnout. These studies32- 35 suggest that a lack of autonomy, difficulty balancing personal and professional life, excessive administrative tasks, and high patient volume are the greatest sources of stress. The way these and other work characteristics affect a given individual is complex and depends on his or her personal responsibilities (eg, relationships, age of children, other interests), personality, health, and enthusiasm for work. Recognizing these variables, there nevertheless appears to be some common themes among physicians and surgeons who experience burnout.

\section{Methodology:- \\ Study design:-}

This study was community base cross section study.

\section{Study area:-}

It was in KSA.

\section{Study population:-}

Surgeon resident and intern.

Sample size:-

100 Surgeon resident and intern. 


\section{Sample technique:-}

We selected our sample by random sampling .

\section{Collecting tool :-}

The Collected of date was done by 3 part questionnaire : about the Personal data, level of knowledge and awareness for stressamong Surgeon resident and intern.

\section{Analysis :}

The data was collected, then clearance and coding was done, also entered data and analysis was done by SPSS .

\section{Result analysis:-}

: The study Result

The study participant's Demographic analysis:

1.1.1 Distribution of the participants by Age groups:

Table (1) shows age group (24-7) was (29\%) \&age group of (more 27 and less 30) old was (32\%) And age group (More 30-34) represented about (39\%) of the total sample.

Table (1):-

Distribution of the participants by Age groups

Variable Frequency Percent

\begin{tabular}{|l|l|l|l|}
\hline Age & $24-27$ & 29 & $29 \%$ \\
\hline & $28-30$ & 32 & $32 \%$ \\
\hline & $31-34$ & 39 & $39 \%$ \\
\hline & Total & 100 & 100.0 \\
\hline
\end{tabular}

Figure 1:- show age variable

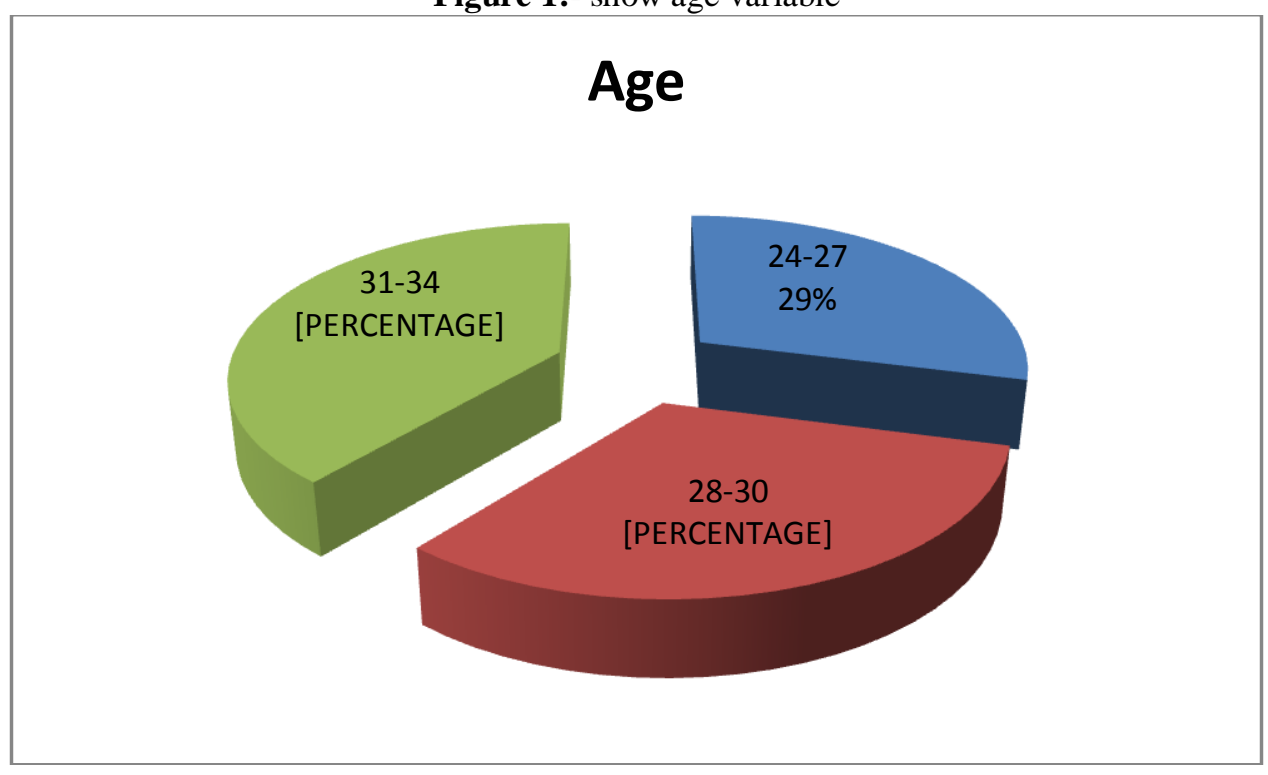

Distribution of the participants by Gender 
Table (2) it shows gender (Male) was the highest (88\%) \& (Female) was (12\%)

Table 2:-

Distribution of the participants by gender

\begin{tabular}{|c|c|c|c|}
\hline Variabl & Frequen & & Percent \\
\hline Gender & Male & 88 & $88 \%$ \\
\hline & Female & 12 & $12 \%$ \\
\hline & Total & 100 & 100.0 \\
\hline
\end{tabular}

Figure 2:- show gender variable

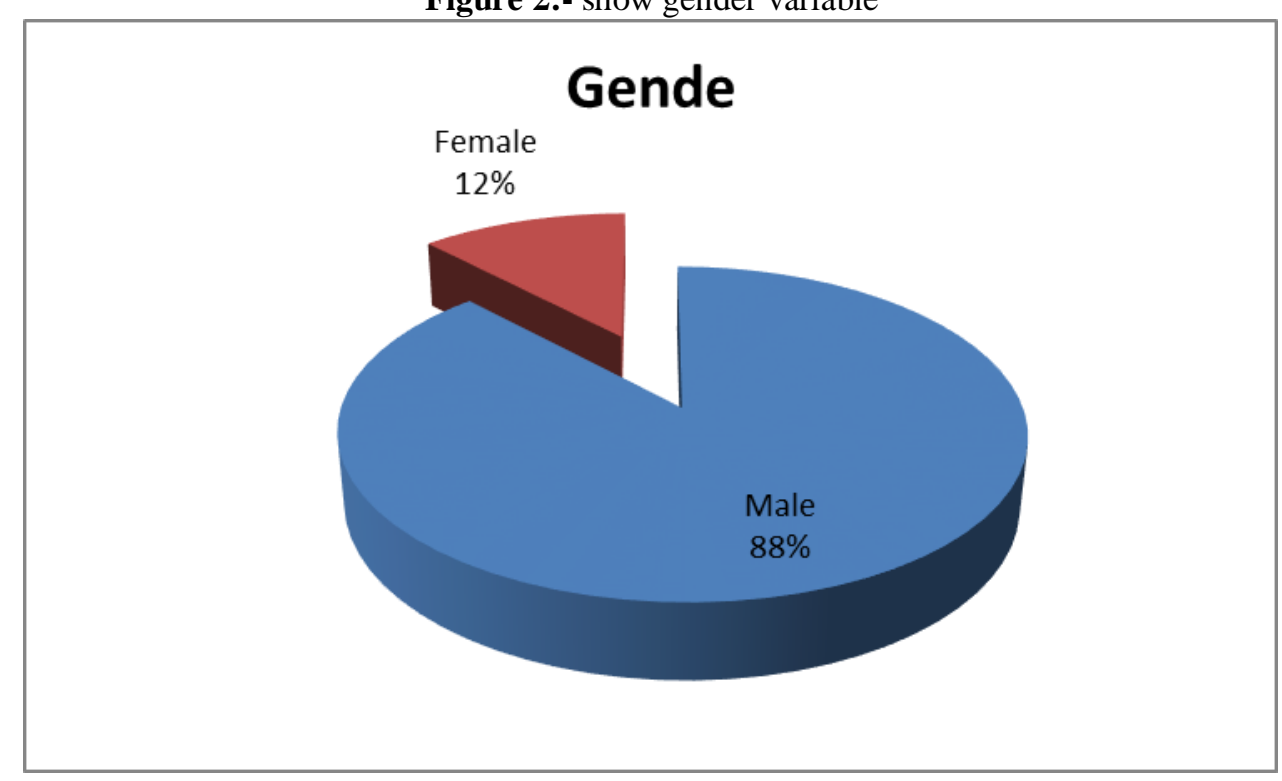

Distribution of the qualification:

Table (3) showed qualification of sample interns and resident, male and intern was $28 \%$ and female was $8 \%$, male and resident was $60 \%$ and female was $4 \%$.

Table 3:-

Shows qualification variable

\begin{tabular}{|l|l|l|l|l|l|l|}
\hline \multicolumn{1}{|c|}{ qualification } & Total & & & & & \\
\hline & intern & percent & resident & percent & intern & \\
\hline Gender & male & 28 & $28 \%$ & 60 & $60 \%$ & 88 \\
\hline & female & 8 & $8 \%$ & 4 & $4 \%$ & 12 \\
\hline Total & 36 & $36 \%$ & 64 & $64 \%$ & 100 & \\
\hline
\end{tabular}


Figure 3:- show qualification variable

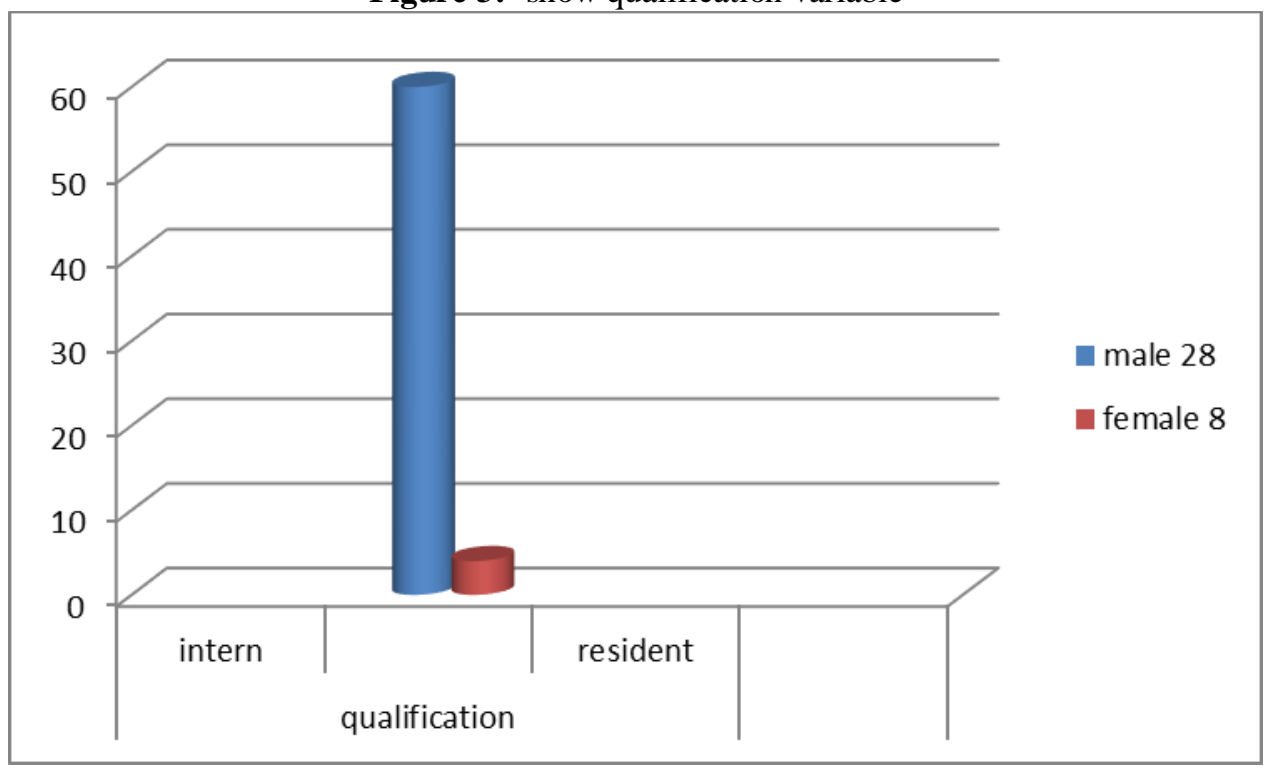

Q1: the prevalence of stress among surgery residents and interns

shows means \& standard deviation for question the average for all question was 3.31 that mean the prevalence stress among surgery residents and interns were in the between fairly often stress and very often.

In the last month how often have you been upset because of something that happened unexpectedly? Mean

\section{Standard Deviation}

In the last month how often have you felt that you were unable to control the important things in your life? 3.79 0.50

In the last month how often have you felt nervous and "stressed"? $3.66 \quad 0.61$

In the last month how often have you felt confident about your ability to handle your personal problems? 3.63 0.60

$\begin{array}{llll}\text { In the last month how often have you felt that things were going your way? } & 2.88 & 1.34\end{array}$

In the last month how often have you found that you could not cope with all the things that you had to do? 3.33 0.82

In the last month how often have you been able to control irritations in your life?3.28 0.83

$\begin{array}{lll}\text { In the last month how often have you felt that you were on top of things? } & 2.89 & 1.21\end{array}$

In the last month how often have you been angered because of things that were outside of your control? $\quad 3.08$ 0.94

In the last month how often have you felt difficulties were piling up so high that you could not overcome them? $3.33 \quad 0.68$

Total $3.31 \quad 0.83$

Q2: explore the causes of stress among surgery residents and interns

questions Mean Standard Deviation

Is being not satisfied about your about your job performanceIs a major source of stress for you $\quad \begin{array}{lll}3.11 & 0.63\end{array}$

Do you think that the reason behind your stress is due to unrealistic Expectaions from either your supervisors or patients?3.24 0.79

Do you feel stressed because financial income is less than expected? $\quad 3.21 \quad 0.83$

Do you think increased responsibility and taking on extra duties during your Shift causes you stress?

0.82

You have too much work to do, and cannot say no to new tasks assigned To you by your supervisor? 0.84

Do you feel that your stress increases when you work in a crowded area?

$3.07 \quad 0.89$

On a personal level, not taking care of your family and yourself like before is a cause of stress for you?

0.82

Total $\quad 3.17 \quad 0.80$ 
Table (4) shows means \& standard deviation for question the average for all question was 3.18 that mean the causes of stress among surgery residents and interns were in the fairly often and the most causes was the "Do you think that the reason behind your stress is due to unrealistic Expectaions from either your supervisors or patients?" it takes the highest mean 3.24, then "Do you feel stressed because financial income is less than expected?" these was the most causes

Q3: explore the methods used to relieve the stress

Table (4) shows means \& standard deviation for question the average fo all question was 2.50 , the most methods used to relieve the stress was "Do you avoid communication or discussion with staff to minimize your stress?" then "Do you think that only solution to your pressure at work is by leaving work in this department?"

Mean Standard Deviation

Do you relieve your stress by sleeping more hours than you did before $2.55 \quad 1.15$

Do you think that ignoring your problems at work without solving them is a the best way to relieve your stress $2.56 \quad 1.17$

Do you think that only solution to your pressure at work is by leaving work in this department? $\quad 2.64 \quad 1.14$

Do you think physical exercise can decrease your stress at work $\quad 2.50 \quad 1.18$

Do you think that good communication and development of your skills at work Will make you more productive and less stressed? $\quad 2.43 \quad 1.14$

$\begin{array}{lll}\text { Do you avoid communication or discussion with staff to minimize your stress? } & 2.68 & 1.05\end{array}$

$\begin{array}{llll}\text { Do you think you can relive your stress by the use of certain medications? } & 2.60 & 1.12\end{array}$

Total $2.57 \quad 1.13$

\section{Conclusions:-}

Physicians pursue the arduous task of becoming surgeons to change the lives of individuals facing serious health problems, to experience the joy of facilitating healing, and to help support those patients for whom medicine does not yet have curative treatments. Despite its virtues, a career in surgery brings significant challenges that can lead to substantial personal distress for the individual surgeon and his or her family. Each surgeon should continuously map a career pathway that integrates personal and professional goals with the outcome of maintaining value, balance, and personal satisfaction throughout his or her professional career. Being proactive in avoiding burnout is preferable to reacting to burnout after it has damaged one's professional life or personal wellness.

There is no single formula for achieving a satisfying professional career. Each of us will have to deal with stressful times in our personal and professional lives; we must cultivate habits of personal renewal, emotional self-awareness, and connection with colleagues and support systems and must find genuine meaning in work to combat these challenges. As surgeons, we also need to set an example of good health. To provide the best care for our patients, we need to be alert, interested in our work, and ready to provide for the patient's needs, which means doing everything we can to stay as healthy as we want our patients to be. Maintaining these values and healthy habits is the work of a lifetime.

\section{Recommendation:-}

- The awareness should be raised about stress among surgery resident and intern.

- Surgery resident and intern need to learn how to avoid, or at least, to lessen stress.

- Surgery resident and intern should never use their job as an excuse for ignoring personal life. This is very important, as physicians tend to deny their personal life and their personal hobbies.

\section{References:-}

1. http://www.ncbi.nlm.nih.gov/pubmed

2. Campbell DAJrSonnadSSEckhauserFECampbellKKGreenfield LJ Burnout among American surgeons. Surgery 2001;130 (4) 696- 705

3. Benson STruskettPGFindlay B The relationship between burnout and emotional intelligence in Australian surgeons and surgical trainees. ANZ J Surg 2007;77 ((suppl 1)) A79

4. Sharma ASharpDMWalker LG et al. Stress and burnout in colorectal and vascular surgical consultants working in the UK National Health Service. Psychooncology 2008;17 (6) 570- 576 
5. KuererHMEberleinTJPollock RE et al. Career satisfaction, practice patterns and burnout among surgical oncologists: report on the quality of life of members of the Society of Surgical Oncology. Ann SurgOncol 2007; 14 (11) 3043- 3053

6. MaslachCJacksonSLeiter M Maslach Burnout Inventory Manual. Palo Alto, CA Consulting Psychologists Press1996

7. Spitzer RLWilliamsJBKroenke $\mathrm{K}$ et al. Utility of a new procedure for diagnosing mental disorders in primary care: the PRIME-MD 1000 study. JAMA 1994;272 (22) 1749- 1756

8. Johnson JTWagnerRLRuegerRMGoepfert H Professional burnout among head and neck surgeons: results of a survey. Head Neck 1993;15 (6) 557- 560

9. BertgesYost WEshelmanARaoufiMAbouljoud MS A national study of burnout among American transplant surgeons. Transplant Proc 2005;37 (2) 1399- 1401

10. RollmanBLMeadLAWangNYKlag MJ Medical specialty and the incidence of divorce. N Engl J Med 1997;336 (11) $800-803$

11. WardeCMMoonesingheKAllenWGelberg L Marital and parental satisfaction of married physicians with children. J Gen Intern Med 1999;14 (3) 157- 165

12. SotileWMSotile MO Physicians' wives evaluate their marriages, their husbands, and life in medicine: results of the AMA-Alliance Medical Marriage Survey. Bull Menninger Clin 2004;68 (1) 39- 59

13. Ford DEMeadLAChangPPCooper-Patrick LWangNYKlag MJ Depression is a risk factor for coronary artery disease in men: the precursors study. Arch Intern Med 1998;158 (13) 1422- 1426

14. Frank EDingle AD Self-reported depression and suicide attempts among U.S. women physicians. Am J Psychiatry 1999;156 (12) 1887- 1894

15. Frank E McMurray JELinzerMElonLSociety of General Internal Medicine Career Satisfaction Study Group, Career satisfaction of US women physicians: results from the Women Physicians' Health Study. Arch Intern Med 1999;159 (13) 1417- 1426

16. Schindler BANovackDHCohen DG et al. The impact of the changing health care environment on the health and well-being of faculty at four medical schools. Acad Med 2006;81 (1) 27- 34

17. Frank EBiolaHBurnett CA Mortality rates and causes among U.S. physicians. Am J Prev Med 2000;19 (3) 155- 159

18. Center CDavisMDetre $\mathrm{T}$ et al. Confronting depression and suicide in physicians: a consensus statement. JAMA 2003;289 (23) 3161- 3166

19. Lindeman SLaaraEHakkoHLonnqvist $\mathrm{J}$ A systematic review on gender-specific suicide mortality in medical doctors. Br J Psychiatry 1996;168 (3) 274- 279

20. Harms BAHeiseCPGouldJCStarling JR A 25-year single institution analysis of health, practice, and fate of general surgeons. Ann Surg 2005;242 (4) 520- 529

21. Hughes PHBrandenburgNBaldwin DC Jr et al. Prevalence of substance use among US physicians. JAMA 1992;267 (17) 2333- 2339

22. AachRDGirardDEHumphrey $\mathrm{H}$ et al. Alcohol and other substance abuse and impairment among physicians in residency training. Ann Intern Med 1992;116 (3) 245- 254

23. Morse RMMartinMASwensonWMNiven RG Prognosis of physicians treated for alcoholism and drug dependence. JAMA 1984;251 (6) 743- 746

24. Shanafelt T A career in surgical oncology: finding meaning, balance, and personal satisfaction. Ann SurgOncol 2008;15 (2) 400- 406

25. ShanafeltTDSloanJAHabermann TM The well-being of physicians. Am J Med 2003;114 (6) 513- 519

26. Spickard AJrGabbeSGChristensen JF Mid-career burnout in generalist and specialist physicians. JAMA 2002;288 (12) 1447- 1450

27. ShanafeltTChungHWhiteHLyckholm LJ Shaping your career to maximize personal satisfaction in the practice of oncology. J ClinOncol 2006;24 (24) 4020- 4026

28. Gabbard GO The role of compulsiveness in the normal physician. JAMA 1985;254 (20) 2926- 2929. 